\section{G86(P) PAEDIATRIC ADVANCED TRAUMA SKILLS (PATS): A NEW ADVANCED TRAUMA COURSE FOR MULTIDISCIPLINARY STAFF}

${ }^{1}$ A Anpananthar, ${ }^{1}$ A Parikh, ${ }^{2} S$ Masud, ${ }^{3} \mathrm{~N}$ Edmonds, ${ }^{4} \mathrm{E}$ Makins. ${ }^{1}$ Paediatric Emergency Medicine, Royal London Hospital, Barts Health NHS Trust, London, UK; ${ }^{2}$ Emergency Medicine, Oxford University Hospital, Oxford, UK; ${ }^{3}$ Paediatric Anaesthesia and PaediatricIntensive Care, Royal London Hospital, Barts Health NHS Trust, London, UK; ${ }^{4}$ Paediatric Surgery, King College Hospital, London, UK

10.1136/archdischild-2015-308599.85

Background Major paediatric trauma is rare and therefore exposure to it is often sporadic and infrequent. Since the designation of major trauma centres (MTC), doctors in these centres are likely to see more paediatric trauma. Advances in paediatric trauma management and are not taught in the more traditional courses such as APLS, ATLS and ETC.

This course has been designed for those with an interest in paediatric trauma based in either MTCs or major trauma units (MTU). There are no other similar courses at present in England.

Method We initially designed a 1-day high fidelity simulation course. Following 8 courses, it became clear there was a need to cover advanced trauma skills. PATS was subsequently developed as a 2-day course covering essential advanced skills. It is run with senior multidisciplinary faculty from emergency medicine, pre-hospital care, paediatric emergency medicine, surgeons, anaesthetists and paediatric intensive care. This high-fidelity simulation course incorporates technical and non-technical skills. The candidates completed anonymous pre- and postquestionnaires.

Results Of the 10 candidates who have attended the 2 courses, many had requested at the start of the course for more experience with complex trauma scenarios, procedures and leading the team. Despite $70 \%$ of the candidates having been involved in other trauma training courses and feeling confident in managing an injured child prior to this course, $90 \%$ found the course positively challenging and 100\% reported that this course had met their expectations.

All candidates had identified human factors as their main learning, in particular communication and team leadership. Other key themes identified were an increased confidence with specific procedures and management protocols. Practicing more procedures during scenarios was requested. Candidates had commented on the benefits of having a mixed seniority of the candidate group (senior trainees and consultants) and the faculty.

Discussion The feedback has been overwhelmingly positive. Evaluation demonstrated the importance of this course for multi-grade and multidisciplinary staff seeing paediatric trauma. Future courses will invite staff from all disciplines exposed to paediatric trauma and will address the request for more practical procedures.

\section{G87(P) PROVIDING SAFER RESUSCITATIONS IN SMALL HOSPITALS AND CLINICAL SITES WITH INFREQUENT RESUSCITATIONS}

R Puttha, A Dev, G Setty, B Pai, M Jain, S Yuen. Paediatrics, George Eliot Hospital, Nuneaton, UK

10.1136/archdischild-2015-308599.86
Aim The aim of our project is to provide safe resuscitations in a small district general hospital (DGH) as measured by the clinical incidents, patient outcomes and staff feedback.

Method Regular multi-disciplinary scenario based resuscitation training sessions were provided, at the point of care. These training cycles focused on the needs of the local team of a small DGH. Regular feedback was obtained from participants. Clinical incidents were analysed for a year before and after the point in time when training was initiated

Results The first Plan-Do-Study-Act (PDSA) cycle of training was delivered along the standard resuscitation training provided in UK Life Support courses. It was immediately obvious that the training required to be more focused towards local needs, which were more extensive compared to that provided in life supportive courses. 3 subsequent PDSA cycles focused on individual and team needs in a multi-disciplinary team such as drills for infusions, setting and using ventilator, airway skills and providing leadership while working in a resident shift. Analysis of the reported clinical incidents, anonymised staff feedback, reflection from resuscitations, personal stories and opinions of the team members - all formed the basis of a series of the next 4 PDSA cycles of resuscitation training. Many of these incorporated simulations of locally performed resuscitation scenarios such as trauma, burns and status epilepticus. The last 4 PDSA cycles were focused on the current needs of a new team, to develop resilience and challenge through human factors and team strategies and tools to enhance performance and patient Safety (TEAM STEPPS). We have at present achieved a steady state.

The staff feedback of these training sessions has been very positive. The clinical incidents of medium and high risk have been reduced by half, while overall incident reporting rate has increased by 1.7 times, promoting an open culture and risk anticipation.

Conclusion Regular multi-disciplinary training focused on the local team and task needs seems to provide safer resuscitations in a small DGH, with a low frequency of resuscitations. This approach might be of use at any clinical site having a potential risk of infrequent paediatric resuscitations.

\section{G88(P) WHICH INTRAVENOUS BRONCHODILATOR? IS LACK OF CLEAR GUIDANCE INCREASING VARIATION IN PRACTICE?}

'L Bentley, ${ }^{2}$ B Azadi, ${ }^{2}$ J Ross. ${ }^{1}$ Faculty of Medicine, Imperial College London, London, UK;

${ }^{2}$ Paediatric Department, Chelsea and Westminster NHS Foundation Trust, London, UK

\subsection{6/archdischild-2015-308599.87}

Aim At present there is not sufficient evidence to suggest the optimal choice, or order, of intravenous bronchodilator in the management of life threatening wheeze or asthma in children. As a result, recent BTS guidelines are unable to give clear advice. This has led to variation at a national and also led to confusion within many departments. We set out to review variations within a group of children aged $>1$ year of age admitted from a busy Paediatric Emergency Department (PED) to a co-located Paediatric HDU.

Methods All admissions from PED to Paediatric HDU with wheeze or an acute exacerbation of asthma requiring intravenous bronchodilators over a 7 month period running from April to November 2014 were identified by review of electronic records. Those $<1$ year of age were excluded from analysis. 